\title{
Introducing the next generation sequencing in genomic amnio and villuos sampling. The so called "Next Generation Prenatal Diagnosis" (NGPD)
}

\author{
Claudio Giorlandino ${ }^{1}$ \\ Alvaro Mesoraca ${ }^{2}$ \\ Domenico Bizzoco² \\ Claudio Dello Russo² \\ Antonella Cima ${ }^{2}$ \\ Gianluca Di Giacomo² \\ Pietro Cignini ${ }^{1}$ \\ Francesco Padula ${ }^{1}$ \\ Nella Dugo ${ }^{1}$ \\ Laura D'Emidio' \\ Cristiana Brizzi ${ }^{1}$ \\ Raffaella Raffio ${ }^{1}$ \\ Vincenzo Milite ${ }^{1}$ \\ Lucia Mangiafico $^{1}$ \\ Claudio Coco ${ }^{1}$ \\ Ornella Carcioppolo ${ }^{1}$ \\ Roberto Vigna ${ }^{1}$ \\ Marialuisa Mastrandrea ${ }^{1}$ \\ Luisa Mobili ${ }^{1}$
}

1 Artemisia Fetal Maternal Medical Centre, Department of Prenatal Diagnosis, Rome, Italy

2 Artemisia Fetal Maternal Medical Centre, Department of Genetics, Rome, Italy

In the last 30 years, invasive prenatal diagnosis has predominantly involved research into chromosomal anomalies, in particular Down's Syndrome (1).

In the last 10 years, parents have been requesting ever more information during pregnancy $(2,3)$ and there has been an increase in the number of cases with ultrasound markers concerning possible fetal complications of unknown origin. This has led to the introduction of prenatal diagnosis and increasingly detailed techniques such as CGH Array (4-6).

These techniques have become standard diagnostic practice in cases where the ultrasound scan provides a conflicting result. However, in reality, such procedures are thought to cover only $10 \%$ of the fetal anomalies linked to genetic malformations discovered at birth (7). Prenatal diagnosis is becoming more and more detailed due to the continual legal action taken by parents regarding diagnostic ultrasound which fails to identify fetal anomalies and regarding unwanted births in general (8-10).

In fact, the continuous evolution of human genetics has led to the development of extremely detailed methodologies, which are able to evaluate not only the errors in chromosomes, both "big errors" (karyotype) and "small errors" (microdeletions, microduplications), but also gene mutations.
To date, approximately 19,000 coding genes contained in the human exome have been identified. The recent introduction of NGS (Next Generation Sequencing) has made it possible, in theory, to explore the entire exome and reveal every form of mutation (11-15).

Therefore, it is possible, today, to open up a completely new diagnostic scenario which would have been considered impossible only a few years ago. However, if this development is not controlled, it could lead to a so-called genetic "deviation", i.e. a genetics that could have unforeseen repercussions on the life and dignity of the individual.

In fact, the risks concerning possible social, emotional and financial consequences in the family and individual is very high. The potential negative impact of prenatal genetic testing must respect the "right not to know". The exaggeration in ever more detailed testing concerning the genetic structure of the embryo creates tension within a family. In the future, this could create genetic discrimination regarding employment or health insurance costs $(16,17)$.

Despite the fact there is theoretically no technical limit to these methodologies, it is important to establish ethical and moral guidelines, at least regarding how these new methodologies are used in prenatal diagnosis.

\section{Technical limits of prenatal diagnostic methodologies}

Prenatal diagnosis, unlike screening, is not simply limited to selecting populations that risk living birth to Down's Syndrome children. In fact, depending on the method used, it can explore all the chromosomal and genetic pathologies that can be diagnosed following birth (18-25).

In fact, the following methods can be used in prenatal diagnosis on a routine basis or in high risk populations:

- traditional cytogenetics, introduced in the 1950s, makes it possible to identify chromosome anomalies, which can be numerical (such as trisomy, monosomy), or structural (translocations, deletions and inversions) (26).

- QF-PCR (Quantitative Fluorescent Polymerase Chain Reaction). This technique which was initially introduced in the USA in 1993, produces a precise and quick diagnosis concerning the most common fetal aneuploidies responsible for the most frequent neonatal pathologies (Down's Syndrome, Patau, Edwards, Turner, Klinefelter) (27).

- Gene sequencing; the first generation of genomic sequencing was developed by Sanger in 1975 (chain-termination method) and by Maxam and 
Gilbert in 1977 (chemical sequencing methods). Sanger's method was found to be technically less complicated and has evolved considerably over the years. The time and costs needed to sequence the DNA represent a limit of this technique (28-30).

- Array-Comparative Genomic Hybridization (CGH) was introduced in 1992 and is based on the comparative genomic hybridization of the patient and a reference genome which is considered normal. In this way, it is possible to identify microdeletions and microduplications (4-6, 31).

- NGS (Next-generation sequencing), which was introduced in 2005, involves the sequencing of DNA molecules amplified clonally or of single molecules of DNA which are spatially separated in flow cells. This strategy represents a radical change compared to Sanger's sequencing method, which is based on the electrophoretic separation of fragments of varying lengths obtained through single sequencing events and which, therefore, has the advantage of reducing time and costs, but above all with this technique it is possible to obtain a considerable quantity of information with one single sequencing cycle (11-15).

\section{Ethical limits concerning prenatal diagnostic methodologies}

One of the consequences regarding the wide range of genetic tests available today is that it is necessary to establish a series of moral, ethical and ideological principles in order to define limits concerning the utilization of these techniques.

The principles that should be taken into consideration are as follows:

- Freedom for the couple to procreate responsibly and to know, in accordance with the rules and regulations established in the country of origin, the state of health of their child.

- The right to life of the foetus in cases where the presence of an altered genetic structure is not serious enough to classify them as wrongful life.

The limits governing the application of these techniques must, therefore, vary depending on the populations examined.

High risk populations (family, maternal age, the presence of genetic markers) can be advised to test one or more specific problems or advised to use all the methodologies currently available, above all if the objective of these interventions is to guarantee the two above-mentioned principles. In particular, regarding the quality of life of the new-born child, some investigations, such as the search for the mutations responsible for congenital deafness or for cystic fibrosis, can make it possible to set up interventions that can improve the outcome of the new-born child (32-34).

In low risk populations, there is an increasing number of couples that, for various reasons, request precise details regarding the health of the foetus. These range from serious situations such as anxiety or social and financial difficulty to less ethical and hedonistic consider- ations. Whatever the underlying reason, these people want to know exactly the state of health of the foetus. Even though this will never be possible, it is however evident that the use of various technologies, such as the ones listed above, in low-risk prenatal diagnosis can identify much more than the $10 \%$ of genetic anomalies that are currently revealed with traditional methods.

Is it now possible, therefore, to offer this population a complete diagnostic test?

In Italy, in 1978 a law came into force which establishes that mothers have the right to obtain all the information that medical science is able to provide regarding the health of their child, so that pregnancy can progress responsibly (35).

The High Court of Cassation, recently stated that, in an important and significant sentence, medics are to be considered entirely responsible should they fail to inform the mother that there are tests which can provide certain diagnoses regarding anomalies that could arise (36).

Medics must, therefore, provide information regarding the availability of sophisticated diagnostic techniques, although they are not obliged to propose or impose their utilization.

What will these ethical limits be?

We are of the opinion that prenatal diagnostic techniques should remain within certain limits and in particular, there should be:

- no investigation into genetic errors which do not provide a clear clinical picture

no investigation into SNPs (single Nucleotide Polymorphisms) which simply indicate a predisposition towards the onset of degenerative diseases or tumors

- no investigation into pathologies that are, however, compatible with a normal or acceptable quality of life, such as diabetes, hypertension and metabolic diseases

- no investigation into diseases that start in later life, such as Alzheimer.

\section{The clinical use of NGS}

Taking into consideration our knowledge regarding genetic diseases, their frequency and the clinical correlation between the alteration of the DNA and resulting pathologies which follow the above-mentioned technical and ethical criteria, a system could be proposed whereby, instead of investigating the 19,000 genes currently known on the exome, investigations could be limited to only 300 of these genes, whose mutations codify for approximately one hundred wellknown and well-defined pathologies (Tab. 1).

Together with these, it is possible to utilize traditional genomic technologies, such as CGH array, in association with NGS (Tab. 2).

Traditional cytogenetic analyses can also be added to these genetic techniques. In fact, these methods can be used to diagnose approximately 350 pathologies, which, being the most frequent, represent more than $80 \%$ of the 6,760 pathologies currently known today (37). 
Introducing the next generation sequencing in genomic amnio and villuos sampling.

The so called "Next Generation Prenatal Diagnosis" (NGPD)

Table 1. Syndromic disorder caused by mutation of genes.

\begin{tabular}{|c|c|c|c|}
\hline Disorder & Transmission & Incidence & Gene \\
\hline Achondrogenesis la & Recessive & $1 / 40000$ & Trip11 \\
\hline Achondrogenesis lb & Recessive & $1 / 40000$ & Dtdst \\
\hline Achondrogenesis li & Dominant & $1 / 40000$ & Col2a1 \\
\hline Acondroplasia & Dominant & $0.5-1 / 10000$ & Fgfr3 \\
\hline Aicardi-Goutieres Syndrome & Recessive & Rare & $\begin{array}{l}\text { Trex; Rnaseh2a; Rnaseh2b; } \\
\text { Rnaseh2c; Samhd1 }\end{array}$ \\
\hline Alpha Talassemia & Recessive & // & $\mathrm{Hba1}$;Hba2 \\
\hline Beta Talassemia & Recessive & // & $\mathrm{Hbb}$ \\
\hline Ambiguous Genitalia & & $1 / 1000$ & Sox9; Wt1; Dax1; Wnt4 \\
\hline Androgen Insensitivity Syndrome & X Linked & $1 / 20000$ & $\operatorname{Ar}$ \\
\hline Angelman & Sporadic & $1 / 12000$ & Ube3a; Snrpn \\
\hline Apert & Sporadic & $1 / 60000$ & Fgfr2 \\
\hline Ataxia Telangectasia & Recessive & $1 / 40000$ & Atm \\
\hline Beckwith Wiedemann & Sporadic & $1 / 13000$ & Cdkn1c; H19; Igf2; Kcnq1ot1 \\
\hline Brugada Syndrome Type 1 & Dominant & $5: 10000$ & Scn5a \\
\hline Cardiomyophaty & & & $\begin{array}{l}\text { Abcc9;Actc1;Acn2; Calr3; } \\
\text { Cav3; Csrp3; Des; Dsg2; Dtna; } \\
\text { Eya4; Fktn; Jph2; Lamp2; } \\
\text { Ldb3; Lmna; Mioz2; Mybpc3; } \\
\text { Myh6; Myh7; Myl2; Myl3; } \\
\text { Mylk2; Nexn; PIn; Prkag2; } \\
\text { Psen1; Psen2; Rbm20; Scn5a; } \\
\text { Sgcd; Slc25a4; Taz; Tcap; } \\
\text { Tmpo; Tnnc1; Tnnt2; Tpm1; } \\
\text { Tnni3; Ttn; Vcl }\end{array}$ \\
\hline Charcot Marie Tooth Cmt1 & Dominant & & $\begin{array}{l}\text { Pmp22 (Cmt1a And Cmt1e); } \\
\text { Mpz (Cmt1b); Litaf (Cmt1c); } \\
\text { Egr2 (Cmt1d); Nefl (Cmt1f) }\end{array}$ \\
\hline Charcot Marie Tooth Cmt2 & Recessive & $1 / 2500$ & $\begin{array}{l}\text { Mfn2; Kif1b (Cmt2a); Rab7a } \\
\text { (Cmt2b); Lmna (Cmt2b1); } \\
\text { Trpv4 (Cmt2c); Bscl2; Gars } \\
\text { (Cmt2d); Nefl (Cmt2e); Hspb1 } \\
\text { (Cmt2f); Mpz (Cmt2i And } \\
\text { Cmt2j); Gdap1 (Cmt2k);Hspb8 } \\
\text { (Cmt2l); Dnm2 }\end{array}$ \\
\hline Charcot Marie Tooth Cmt4 & Recessive & & $\begin{array}{l}\text { Gdap1 (Cmt4a); Mtmr2 } \\
\text { (Cmt4b1); Sbf2 (Cmt4b2); } \\
\text { Sh3tc2 (Cmt4c); Ndrg1 } \\
\text { (Cmt4d); Egr2 (Cmt4e); Prx } \\
\text { (Cmt4f); Fgd4 (Cmt4h); Fig4 } \\
\text { (Cmt4j) }\end{array}$ \\
\hline Charcot Marie Tooth Cmtx & X Linked & & Gjb1 (Cmtx1); Prps1 (Cmtx5) \\
\hline Charge Syndrome & Dominant & $1 / 10000$ & Chd7 \\
\hline Ciliary Dyskinesia & Recessive & $1 / 16000$ & Dnai1and Dnah5 \\
\hline Congenital Adrenal Hyperplasia & Recessive & $1 / 12000$ & Cyp21a2 \\
\hline Congenital Hypothyroidism & Sporadic & $1 / 4000$ & $\begin{array}{l}\text { Duox2; Pax8; Slc5a5; Tg; Tpo; } \\
\text { Tshb; Tshr }\end{array}$ \\
\hline
\end{tabular}

to be continued 
Continued from Table 1.

\begin{tabular}{|c|c|c|c|}
\hline Disorder & Transmission & Incidence & Gene \\
\hline Cystic Fibrosis & Recessive & $1 / 2500$ & Cftr \\
\hline De Lange Syndrome & Dominant & $1 / 10000$ & Nipbl; Smc3 \\
\hline De Lange Syndrome & X Linked & $1 / 10000$ & Smc1a \\
\hline Early-Onset Primary Dystonia & Dominant & $1 / 10000$ & Tor1a \\
\hline Hereditary Elliptocytosis Type 1 & Dominant & $1 / 10000$ & Epb41 \\
\hline $\begin{array}{l}\text { Congenital Isolated Thyroxine-Binding } \\
\text { Globulin Deficiency }\end{array}$ & Dominant X-Linked & $1 / 2000$ & Serpina7 \\
\hline Dystrophinopathies & X Linked Recessive & $1 / 3500$ & Dmd \\
\hline Ehlers-Danlos Syndrome & Dominant/Recessive & $1 / 5000$ & $\begin{array}{l}\text { Adamts2; Col1a1; Col1a2; } \\
\text { Col3a1; Col5a1; Col5a2; Plod1; } \\
\text { Tnxb }\end{array}$ \\
\hline Ellis Van Creveld & Recessive & $1 / 5000$ & Evc1; Evc2 \\
\hline Epidermolysis Bullosa & Recessive & $1 / 30000$ & Krt5; Krt14; Col7a1; Plec \\
\hline $\begin{array}{l}\text { Facioscapulohomeral } \\
\text { Muscular Dystrophy }\end{array}$ & Dominant & $1 / 20000$ & Fshd1 \\
\hline Familial Mediterranean Fever & Recessive & $1 / 1000$ & Mefv \\
\hline Fanconi Anemia & Recessive & $1 / 160000$ & Fanca; Fancc; Fancg \\
\hline Fetal Akinesia Deformation Sequence & Sporadic & $1 / 12000$ & $\begin{array}{l}\text { Chrna1; Chrnb1; Chrnd; } \\
\text { Rapsn; Dok7 }\end{array}$ \\
\hline Galactosemia & Recessive & $1 / 30000$ & Galt \\
\hline Gaucher Disease & Recessive & $1 / 10000$ & Gba \\
\hline $\begin{array}{l}\text { Glucose-6-Phosphate } \\
\text { Dehydrogenase Deficiency }\end{array}$ & X Linked & ?? & G6pd \\
\hline Glycogen Storage Disease Type li & Recessive & $1 / 50000$ & Gaa \\
\hline Gorlin Syndrome & Dominant & $1 / 30000$ & Ptch1 \\
\hline Hemophilia A & X Linked Recessive & $1 / 5000$ & Fviii \\
\hline Hereditary Hemochromatosis & Recessive & $1 / 500$ & $\mathrm{Hfe}$ \\
\hline Hereditary Multiple Exostoses & Dominant & $1 / 50000$ & Ext1; Ext2 \\
\hline Hirschsprung & Dominant & $1 / 10000$ & Edn3; Ednrb; Ret \\
\hline Holoprosencephaly & Sporadic & $1 / 16000$ & $\begin{array}{l}\text { Hpe; Shh; Zic2; Gli2; Fast1; } \\
\text { Ptch; Dhcr7; Disp1; Nodal; } \\
\text { Foxh1; Fgf8 }\end{array}$ \\
\hline Holoprosencephaly Nonsyndromic & Dominant & $1 / 10000$ & Shh; Zic2; Six3 \\
\hline Hypochondroplasia & Sporadic & $1 / 15000-40000$ & Fgfr3 \\
\hline Hypohidrotic Ectodermal Dysplasia & X Linked & $1 / 10000$ & Eda1 \\
\hline Kabuki & $\begin{array}{l}\text { Dominant }(\mathrm{Kmtd} 2 \mathrm{~d}) / \\
\text { X-Linked Dominant } \\
(\mathrm{Kdm6a})\end{array}$ & $1 / 32000$ & Kmt2d; Kdm6a \\
\hline Long Qt Syndrome (Lqt1-12) & Dominant & $1 / 7000$ & $\begin{array}{l}\text { Kcnq1; Kcnh2; Scn5a; Ank2; } \\
\text { Kcne1; Kcne2; Kcnj2; Cacna1c; } \\
\text { Cav3; Scn4b; Akap9; Snta1 }\end{array}$ \\
\hline Marfan Syndrome & Dominant/Sporadic & $1 / 10000$ & Fbn1 \\
\hline Metachromatic Leukodystrophy & Recessive & $1 / 40000$ & Arsa; Psap \\
\hline
\end{tabular}


Introducing the next generation sequencing in genomic amnio and villuos sampling.

The so called "Next Generation Prenatal Diagnosis" (NGPD)

Continued from Table 1.

\begin{tabular}{|c|c|c|c|}
\hline Disorder & Transmission & Incidence & Gene \\
\hline Microcephaly & Recessive & $1 / 30000-200000$ & Aspm \\
\hline Mucopolysaccharidosis Type 1 & Recessive & $1 / 100000$ & Idua \\
\hline Multiple Endocrine Neoplasia Type 1 & Dominant & $1 / 30000$ & Men1; Ret; Cdkn1b \\
\hline Multiple Epiphyseal Dysplasia & Dominant / Sporadic & $1 / 10000$ & $\begin{array}{l}\text { Comp; Col9a1; Col9a2; } \\
\text { Col9a3; Matn3 }\end{array}$ \\
\hline Nail-Patella Syndrome & Dominant & $1 / 50000$ & $\operatorname{Lmx} 1 b$ \\
\hline Neural Tube Defects & Sporadic & $1 / 500$ & Mthfr \\
\hline Neurofibromatosis I & Dominant & $1 / 3000$ & Nf1 \\
\hline Neurofibromatosis II & Dominant & $1 / 25000$ & $\mathrm{Nf} 2$ \\
\hline $\begin{array}{l}\text { Neuromuscular Disorders-Congenital } \\
\text { Muscular Dystrophies }\end{array}$ & & & $\begin{array}{l}\text { Acta1; Ampd1; Ampd3; Ano5; } \\
\text { Capn3; Cav3; Col6a1; Col6a2; } \\
\text { Col6a3; Des; Dmd; Dysf; Emd; } \\
\text { Fkrp; Fktn; Itga7; Lama2; } \\
\text { Large; Lmna; Myot; Neb; Pex1; } \\
\text { Pex12; Pex14; Pex2; Pex26; } \\
\text { Pex3; Pex5; Pex6; Plec; } \\
\text { Pmm2; Pomgnt1;Pomt1; } \\
\text { Pomt2; Ryr1; Ryr2; Sepn1; } \\
\text { Sgca; Sgcb; Sgcd; Sgce; Sgcg; } \\
\text { Sil1; Tcap; Tnni2; Tnnt1; Tpm2; } \\
\text { Tpm3; Trim32; Ttn }\end{array}$ \\
\hline $\begin{array}{l}\text { Noonan, Leopard, Costello } \\
\text { And Cardiofaciocutaneous Syndrome }\end{array}$ & & $\begin{array}{l}1 / 1000-2500 \\
\text { (Noonan) }\end{array}$ & $\begin{array}{l}\text { Ptpn11; Sos1; Kras; Raf1; Braf; } \\
\text { Mek1; Nraf; Map2k1; Map2k2; } \\
\text { Hras; Nras; Cbl; Shoc2 }\end{array}$ \\
\hline Oral-Facial-Digital Syndrome & X Linked Dominant & $1 / 50000$ & Ofd1 \\
\hline Dissecans Osteochondritis & Dominant & $1 / 3000$ & Acan \\
\hline Osteogenesis Imperfecta & Dominant & $1 / 10000$ & Col1a1; Col1a2 ; Crtap; Lepre1 \\
\hline Osteopoikilosis & Dominant & $1 / 50000$ & Lemd3 \\
\hline Phenylketonuria & Recessive & $1 / 15000$ & Pah \\
\hline Isolated Pierre Robin Sequence & Sporadic & $1 / 8500$ & Sox9 \\
\hline Polycystic Kidney Disease & Dominant/Recessive & $1 / 4000-10000$ & Pkd1; Pkd2; Pkhd1 \\
\hline Rendu-Osler-Webwr Disease & Acvrl1 & $1 / 10000$ & Acvrl1; Eng; Smad4 \\
\hline Rett Syndrome & X Linked & $1 / 10000$ & Мecp2 \\
\hline Saethre Chotzen & Dominant & $1 / 25000$ & Twist1 \\
\hline Seckel Syndrome & Recessive & $1 / 10000$ & Atr \\
\hline Hereditary Spherocytosis & $\begin{array}{l}\text { Dominant/ Rarely } \\
\text { Recessive }\end{array}$ & $1 / 5000$ & Ank1 \\
\hline Short Qt Syndrome & Dominant & Unknown & $\begin{array}{l}\text { Cacna1b; Cacna1c; Kcnh2; } \\
\text { Kcnj2; Kcnq1 }\end{array}$ \\
\hline Sickle Cell Disease & Recessive & $? ?$ & $\mathrm{Hbb}$ \\
\hline Smith Lemli Opitz Syndrome & Sporadic & $1 / 20000$ & Dhcr7 \\
\hline Sotos Syndrome & Sporadic & $1 / 10000$ & Nsd1 \\
\hline Stickler Syndrome & Dominant/Sporadic & $1 / 7,500$ & $\begin{array}{l}\text { Col2a1; Col11a1; Col11a2; } \\
\text { Col9a1; Col9a2 }\end{array}$ \\
\hline Tay Sachs & Recessive & 1/3600 (Ashkenazi) & Hexa \\
\hline
\end{tabular}


Continued from Table 1.

\begin{tabular}{llll}
\hline Disorder & Transmission & Incidence & Gene \\
\hline Thrombocytopenia-Absent Radius & Sporadic & $1 / 100000$ & Rbm8a \\
Treacher Collins Syndrome & Dominant & $1 / 50000$ & Polr1c; Polr1d; Tcof1 \\
Tuberous Sclerosis & Dominant & $1 / 6000$ & Tsc1; Tsc2 \\
Vacterl Association & Sporadic & $1 / 10000$ & Foxf1; Mthfsd; Foxc2; Foxl1 \\
Von Hippel Lindau & Dominant & $1 / 45000$ & Vhl \\
Von Willebrand Disease & Dominant/ Recessive & $1 / 10000-100000$ & Vwf \\
Waardenburg Syndrome & Dominant & $1 / 40000$ & Edn3; Ednrb; Mitf; Pax3; Snai2; \\
X-Linked Agammaglobulinemia & X Linked & $1 / 200000$ & Sox10 \\
\hline
\end{tabular}

Table 2. Syndromic disorder caused by microdelection/microduplication of genetic locus.

\begin{tabular}{|c|c|c|c|c|c|c|c|}
\hline 1 & $\begin{array}{l}\text { Miller-Dieker Syndrome - } \\
\text { Gene LIS1 - 17p13.3 }\end{array}$ & 51 & $\begin{array}{l}\text { Joubert Syndrome } \\
\text { Type } 4 \text { - 2q13 }\end{array}$ & 101 & Monosomy16p11.2p12.2 & 151 & $\operatorname{Del}(16)(P 11.2)$ \\
\hline 2 & $\begin{array}{l}\text { Autism X-Linked - } \\
\text { Gene NLGN4 - Xp22.33 }\end{array}$ & 52 & $\begin{array}{l}\text { Metachromatic } \\
\text { Leukodystrophy - } \\
22 q 13.33\end{array}$ & 102 & Monosomy16q24.3 & 152 & $\operatorname{Del}(16)(Q 24.3)$ \\
\hline 3 & $\begin{array}{l}\text { Axenfeld-Rieger } \\
\text { Syndrome - Geni PITX2/ } \\
\text { FOXC1 - 4q25-Q26 }\end{array}$ & 53 & $\begin{array}{l}\text { Buschke-Ollendorff } \\
\text { Syndrome - 12q14.2-Q15 }\end{array}$ & 103 & Monosomy17p13.3 & 153 & $\begin{array}{l}\operatorname{Del}(16) \\
(\mathrm{P} 11.2 \mathrm{p} 12.2)\end{array}$ \\
\hline 4 & $\begin{array}{l}\text { Sex-Determining Region } \\
\text { Y - Gene SRY - Yp11.3 }\end{array}$ & 54 & $\begin{array}{l}\text { Microdelection Syndrome } \\
1 \mathrm{q} 21.1\end{array}$ & 104 & Monosomy17q21.31 & 154 & $\operatorname{Del}(16)(P 13.11)$ \\
\hline 5 & $\begin{array}{l}\text { Beckwith-Wiedemann } \\
\text { Syndrome - 11p15.5 }\end{array}$ & 55 & $\begin{array}{l}\text { Microdelection Syndrome } \\
\text { 3q29 }\end{array}$ & 105 & Monosomy17q23.1q23.2 & 155 & $\operatorname{Del}(17)(Q 21.31)$ \\
\hline 6 & $\begin{array}{l}\text { Potocki-Shaffer Syndrome } \\
-11 p 11.2\end{array}$ & 56 & $\begin{array}{l}\text { Microdelection Syndrome } \\
15 q 13.3\end{array}$ & 106 & Monosomy19p13.12 & 156 & $\operatorname{Del}(17)(Q 11)$ \\
\hline 7 & $\begin{array}{l}\text { Prader Willi /Angelman } \\
\text { Syndrome - 15q11-Q13 }\end{array}$ & 57 & $\begin{array}{l}\text { Microdelection Syndrome } \\
17 q 21.31\end{array}$ & 107 & Monosomy19q13.1 & 157 & $\operatorname{Del}(17)(Q 12)$ \\
\hline 8 & $\begin{array}{l}\text { Cat Eye Syndrome - } \\
\text { Geni CECR1, CECR5, } \\
\text { CECR6 - 22q11 }\end{array}$ & 58 & $\begin{array}{l}\text { Delection Syndrome } \\
22 q 11.2 \text { Distal }\end{array}$ & 108 & Monosomy20p12.3 & 158 & $\begin{array}{l}\operatorname{Del}(17) \\
(Q 23.1 q 23.2)\end{array}$ \\
\hline 9 & $\begin{array}{l}\text { Rieger Syndrome - } \\
14 q 25-Q 26\end{array}$ & 59 & Aniridia - $11 p 13$ & 109 & Monosomy20q13.33 & $\begin{array}{l}159 \\
\text { (P13 }\end{array}$ & $\begin{array}{l}6 \operatorname{Del}(19) \\
.12)\end{array}$ \\
\hline 10 & $\begin{array}{l}\text { Charcot-Marie-Tooth } \\
\text { Disease Type } 1 \text { - 17p11.2 }\end{array}$ & 60 & $\begin{array}{l}\text { Charge Syndrome - } \\
8 q 12.2\end{array}$ & 110 & $\begin{array}{l}\text { Monosomy } \\
\text { 21q22.11q22.12 }\end{array}$ & 160 & $\begin{array}{l}\operatorname{Del}(19) \\
(Q 13.11)\end{array}$ \\
\hline 11 & $\begin{array}{l}\text { Charcot-Marie-Tooth } \\
\text { Syndrome X-Linked - } \\
1 \text { Xq13.1 }\end{array}$ & 61 & $\begin{array}{l}\text { Micrioftalmic Syndrome } \\
\text { Type } 7 \text { - Xp22.2 }\end{array}$ & 111 & $\begin{array}{l}\text { Monosomy } \\
21 q 22.13 q 22.2\end{array}$ & 161 & $\operatorname{Del}(20)(P 12.3)$ \\
\hline 12 & $\begin{array}{l}\text { Rubinstein-Taybi } \\
\text { Syndrome - 16p13.3 }\end{array}$ & $\begin{array}{l}62 \\
\text { Syn }\end{array}$ & $\begin{array}{l}\text { Severe Polycystic Kidney } \\
\text { drome - 16p13.3 }\end{array}$ & 112 & $\begin{array}{l}\text { Monosomy22q11.2 } \\
\text { Distale }\end{array}$ & 162 & $\operatorname{Del}(20)(P 13)$ \\
\hline 13 & $\begin{array}{l}\text { Saethre-Chotzen } \\
\text { Syndrome - 7p21 }\end{array}$ & 63 & $\begin{array}{l}\text { Simpolidattilia Type } 1 \text { - } \\
2 q 31.1\end{array}$ & 113 & Dup(1)(Q21.1) & 163 & $\begin{array}{l}1 \mathrm{Del}(20) \\
(\mathrm{Q} 13.33)\end{array}$ \\
\hline 14 & $\begin{array}{l}\text { Cleidocranial Dysplasia - } \\
6 \text { p21 }\end{array}$ & 64 & $\begin{array}{l}\text { Velocardiofacial } \\
\text { syndrome - 22q11.21 }\end{array}$ & 114 & Dup(2)(Q23.1) & 164 & Duplication Xp22 \\
\hline 15 & $\begin{array}{l}\text { Cornelia De Lange } \\
\text { Syndrome - 5p13.1 }\end{array}$ & 65 & Wilms' Tumor- $11 \mathrm{p} 13$ & 115 & Dup(2)(Q31.1) & $\begin{array}{l}165 \\
(Q 22\end{array}$ & $\begin{array}{l}\operatorname{Del}(21) \\
2.13 q 22.2)\end{array}$ \\
\hline
\end{tabular}


Introducing the next generation sequencing in genomic amnio and villuos sampling.

The so called "Next Generation Prenatal Diagnosis" (NGPD)

Continued from Table 2.

\begin{tabular}{|c|c|c|c|c|c|c|c|}
\hline 16 & $\begin{array}{l}\text { Simpson-Golabi-Behmel } \\
\text { Syndrome - Xq26 }\end{array}$ & 66 & Monosomy1p21.3 & 116 & Dup(3)(Q26) & 166 & $\begin{array}{l}\operatorname{Del}(21) \\
(Q 22.13 q 22.2)\end{array}$ \\
\hline 17 & $\begin{array}{l}\text { Cri Du Chat Syndrome - } \\
5 p 15.2\end{array}$ & 67 & Monosomy1q21.1 & 117 & 5 Dup(5)(Q35) & 167 & $\operatorname{Del}(\mathrm{X})(\mathrm{P} 21)$ \\
\hline 18 & $\begin{array}{l}\text { Smith-Magenis Syndrome - } \\
17 p 11.2\end{array}$ & 68 & Monosomy1q41q42 & 118 & Dup(7)(P22.1) & 168 & $\operatorname{Del}(\mathrm{X})(\mathrm{P} 23)$ \\
\hline 19 & $\begin{array}{l}\text { Dandy-Walker Syndrome - } \\
\text { Gene ZIC1-ZIC4 - 3q24 }\end{array}$ & 69 & Monosomy2p15p16.1 & 119 & 6 Dup(8)(P23.1) & 169 & $\begin{array}{l}\text { Telomeric } \\
\text { Duplication Xq }\end{array}$ \\
\hline 20 & Sotos Syndrome - 5q35 & 70 & Monosomy2p21 & 120 & Dup(10)(Q22.3q23.3) & 170 & Duplication Xp22 \\
\hline 21 & $\begin{array}{l}\text { Digeorge Syndrome - } \\
22 q 11.2\end{array}$ & 71 & Monosomy2q23.1 & 121 & Dup(14)(Q11.2) & & \\
\hline 22 & $\begin{array}{l}\text { Digeorge Syndrome } \\
\text { Region } 2 \text { - 10p14-P13 }\end{array}$ & 72 & Monosomy2q24 & 122 & $\operatorname{Dup}(11) P(15.4)$ & & \\
\hline 23 & $\begin{array}{l}\text { Split-Hand/Foot } \\
\text { Malformation } 3 \text { - 10q24 }\end{array}$ & 73 & Monosomy2q32 & 123 & Dup(15)(Q11q13) & & \\
\hline 24 & $\begin{array}{l}\text { Split-Hand/Foot } \\
\text { Malformation } 4 \text { - 3q27 }\end{array}$ & 74 & Monosomy3q13 & 124 & Dup(16)(P13.11) & & \\
\hline 25 & $\begin{array}{l}\text { Split-Hand/Foot } \\
\text { Malformation } 5 \text { - 2q31 }\end{array}$ & 75 & Monosomy3q29 & 125 & $\operatorname{Dup}(17)(\mathrm{P} 13.3)$ & & \\
\hline 26 & $\begin{array}{l}\text { Early Onset Alzheimer's } \\
\text { Disease - 21q21 }\end{array}$ & 76 & Monosomy4q21 & 126 & Dup(17)(Q21.31) & & \\
\hline 27 & $\begin{array}{l}\text { Sinpolidattilia/Sindattilia } \\
\text { Type II - 2q31-Q32 }\end{array}$ & 77 & Monosomy5q14.3 & 127 & Dup(22)(Q11.2) Distal & & \\
\hline 28 & $\begin{array}{l}\text { Feingold Syndrome - } \\
2 \mathrm{p} 24.1\end{array}$ & 78 & Monosomy5q31.3 & 128 & $\operatorname{Dup}(\mathrm{X})(\mathrm{P} 22.13 p 22.2)$ & & \\
\hline 29 & Greig Syndrome - 7p13 & 79 & Monosomy6p22 & 129 & Dup(X)(Q12-Q13.3) & & \\
\hline 30 & $\begin{array}{l}\text { Van Der Woude } \\
\text { Syndrome - 1q32-Q41 }\end{array}$ & 80 & Monosomy6q16 & 130 & $\operatorname{Dup}(X)(Q 27.3 q 28)$ & & \\
\hline 31 & $\begin{array}{l}\text { WAGR Syndrome - } \\
11 \mathrm{p} 13\end{array}$ & 81 & Monosomy7q11.23 & 131 & Duplication 22q11.2 & & \\
\hline 32 & $\begin{array}{l}\text { Holoprosencephaly } \\
\text { Type } 1 \text { - 21q22.3 }\end{array}$ & 82 & Monosomy7q31 & 132 & $\operatorname{Del}(1)(P 36)$ & & \\
\hline 33 & $\begin{array}{l}\text { Holoprosencephaly } \\
\text { Type } 2 \text { - 2p21 }\end{array}$ & 83 & Monosomy8p11.2 & 133 & $\operatorname{Del}(1)(Q 21)$ & & \\
\hline 34 & $\begin{array}{l}\text { Holoprosencephaly } \\
\text { Type } 3 \text { - 7q36 }\end{array}$ & 84 & Monosomy8p23.1 & 134 & $\operatorname{Del}(2)(Q 23.1)$ & & \\
\hline 35 & $\begin{array}{l}\text { Williams Syndrome - } \\
7 q 11.23\end{array}$ & 85 & Monosomy8q13 & 135 & Del(2)(Q32) & & \\
\hline 36 & $\begin{array}{l}\text { Wolf-Hirschhorn } \\
\text { Syndrome - Gene WHSC - } \\
4 \text { p16.3 }\end{array}$ & 86 & Monosomy8q21.11 & 136 & $\operatorname{Del}(2)(Q 37)$ & & \\
\hline 37 & $\begin{array}{l}\text { Lissencephaly X-Linked - } \\
\text { Xq22.3-Q23 }\end{array}$ & 87 & Monosomy8q24.1 & 137 & $\operatorname{Del}(3)(Q 13)$ & & \\
\hline 38 & $\begin{array}{l}\text { Discondrosteosi Di Leri } \\
\text { Weill - Xpter-P22.32 }\end{array}$ & 88 & Monosomy9q22.3 & 138 & $\operatorname{Del}(3)(Q 29)$ & & \\
\hline 39 & $\begin{array}{l}\text { Kallmann Syndrome } \\
\text { Type 1- Gene KAL1 - } \\
\text { Xp22.3 }\end{array}$ & 89 & Monosomy10p11.21p12.31 & 139 & $\operatorname{Del}(4)(Q 21)$ & & \\
\hline
\end{tabular}


Continued from Table 2.

\begin{tabular}{|c|c|c|c|c|c|}
\hline 40 & $\begin{array}{l}\text { Kallmann Syndrome } \\
\text { Type 2-Gene KAL2 - } \\
\text { 8p11.2-P11.1 }\end{array}$ & 90 & Monosomy10q22.3q23.3 & 140 & $\operatorname{Del}(5)(Q 14.3)$ \\
\hline 41 & $\begin{array}{l}\text { Terminal Delection } \\
\text { Syndrome } 14 q \\
\text { (Van Karnebeek) }\end{array}$ & 91 & Monosomy11p13 & 141 & $6 \operatorname{Del}(6)(P 22)$ \\
\hline 42 & $\begin{array}{l}\text { Delection 1p36 } \\
\text { (Monosomy 1p36) }\end{array}$ & 92 & Monosomy12p12.1 & 142 & $\operatorname{Del}(6)(Q 16)$ \\
\hline 43 & Monosomy2q37 & 93 & Monosomy12q15q21.1 & 143 & $6 \operatorname{Del}(6)(Q 25)$ \\
\hline 44 & $\begin{array}{l}\text { Langer Giedion } \\
\text { Syndrome - } \\
\text { 8q24.11-Q24.13 }\end{array}$ & 94 & Monosomy13q32 & 144 & $\operatorname{Del}(7)(Q 31)$ \\
\hline 45 & $\begin{array}{l}\text { Trico-rino-falangea } \\
\text { Syndrome - 8q24.1 }\end{array}$ & 95 & Monosomy14q11.2 & 145 & \\
\hline 46 & $\begin{array}{l}\text { Jacobsen Syndrome - } \\
\text { 11q23.1-Q24.1 }\end{array}$ & 96 & Monosomy14q22q23 & 146 & $\operatorname{Del}(12)(\mathrm{P} 12.1)$ \\
\hline 47 & $\begin{array}{l}\text { Branchio-oto-renal } \\
\text { Syndrome - 8q13.3 }\end{array}$ & 97 & Monosomy14q22-Q23 & 147 & $\operatorname{Del}(13)(Q 14)$ \\
\hline 48 & $\begin{array}{l}\text { Campomelic dysplasia - } \\
17 q 24.3\end{array}$ & 98 & Monosomy $15 q 11.2$ & 148 & $\operatorname{Del}(13)(Q 34)$ \\
\hline 49 & $\begin{array}{l}\text { Cornelia De Lange } \\
\text { Syndrome - } 5 p 13.2\end{array}$ & 99 & Monosomy15q13.3 & 149 & $\operatorname{Del}(14)(Q 12)$ \\
\hline 50 & Johanson-Blizzard & 100 & Monosomy16p11.2 & 150 & $\operatorname{Del}(15)(Q 14)$ \\
\hline
\end{tabular}

On the basis of what we know today, this type of system would be able to cover almost all the pathologies that occur in less than 1 case for every 50,000. Therefore, it becomes very unlikely that the gynaecologist or sonographer can make a mistake in the diagnosis or discover, at birth, the presence of an unexpected pathology.

In fact, the introduction of such a technique in the future could guarantee that couples receive precise information and also that medics could be "protected" from "incidents" where professional responsibility would be involved.

Such important prenatal investigations, however, cannot disregard an accurate and complete genetic consultation, which not only provides parents information regarding diagnostic certainties but also the uncertainties and doubts which can arise from such ample molecular investigations (despite the fact the selection of genes and mutations to be analysed could be limited).

\section{Final considerations}

There is considerable innovation and relevant confusion regarding the world of prenatal genetic testing at the moment.

While on the one hand, the recent introduction of non-invasive tests through the research of foetal DNA on maternal blood is reducing the field of investigation to the screening of only a few aneuploids which offer no guarantees, on the other, there is a low but progressive growth of studies carried out directly on the foetal DNA through invasive techniques.

Therefore, we are heading in two seemingly opposing directions towards unreliable tests which provide limited information on the one side and towards precise tests for an excessive quantity of information on the other. Which direction should we take? Which category of patients should be directed one way and which should be directed in the other?

While for high-risk populations such as those where NIPT or an ultrasound scan reveals a possible anomaly, there seems to be general consensus towards the use of invasive genomic testing, considerable doubt remains regarding low-risk populations.

In this larger latter category, of particular importance are the expectations of the couple, the correct information provided by medics and, above all, the legal medical implications.

In Italy, the Civil Supreme Court has twice convicted medics for having proposed screening tests instead of diagnostic testing (36).

This has generated great interest in obtaining compensation for any diagnosis of a genetic disease considered to be responsible for a "wrongful life" which "potentially" could have been discovered using the scientific methods currently available.

Therefore, the Legislator, in Italy, has practically told the personal gynaecologist not to accept responsibility regarding recommendations to their colleague concerning screening tests for Down's Syndrome. A con- 
Introducing the next generation sequencing in genomic amnio and villuos sampling.

The so called "Next Generation Prenatal Diagnosis" (NGPD)

sequence of this is that they are effectively "obliging" them to propose genetic diagnostic tests. In other words, to guarantee themselves from a legal point of view, they inform the expectant mother of the existing differences in various strategies and they request very precise consensus from the parent.

\section{References}

1. Abu-Rustum RS, Daou L, Abu-Rustum SE. Role of first-trimester sonography in the diagnosis of aneuploidy and structural fetal anomalies. J Ultrasound Med. 2010 Oct; 29(10):1445-1452.

2. Renna MD, Pisani P, Conversano F, Perrone E, Casciaro E, Renzo GC, Paola MD, Perrone A, Casciaro S. Sonographic markers for early diagnosis of fetal malformations. World J Radiol. 2013 Oct 28; 5(10):356-371.

3. Chasen ST, Razavi AS. Echogenic intracardiac foci: disclosure and the rate of amniocentesis in low-risk patients. Am J Obstet Gynecol. 2013 Oct; 209(4):377.

4. Cheung SW, Shaw CA, Yu W, Li J, Ou Z, Patel A, Yatsenko SA, Cooper ML, Furman P, Stankiewicz P, Lupski JR, Chinault AC, Beaudet AL. Development and validation of a CGH microarray for clinical cytogenetic diagnosis. Genet Med. 2005 Jul-Aug; 7(6):422-432.

5. Cignini P, Dugo N, Giorlandino C, Gauci R, Spata A, Capriglione S, Cafà EV. Prenatal diagnosis of a fetus with a ring chromosome 20 characterized by array-CGH. J Prenat Med. 2012 Oct; 6(4):72-73.

6. Cignini P, Dinatale A, D'Emidio L, Giacobbe A, Pappalardo EM, Ermito S, Bizzoco D, Di Giacomo G, Gabrielli I, Mesoraca A, Giorlandino M, Giorlandino C. Prenatal Diagnosis of a Fetus with de novo Supernumerary Ring Chromosome 16 Characterized by Array Comparative Genomic Hybridization. AJP Rep. 2011 Sep; 1(1):29-32. doi: 10.1055/s-00311274512. Epub 2011 Mar 18.

7. Carss KJ, Hillman SC, Parthiban V, McMullan DJ, Maher ER, Kilby MD, Hurles ME. Exome sequencing improves genetic diagnosis of structural fetal abnormalities revealed by ultrasound. Hum Mol Genet. 2014 Feb 11.

8. Frati $P$, Gulino M, Turillazzi E, Zaami S, Fineschi V. The physician's breach of the duty to inform the parent of deformities and abnormalities in the foetus: "wrongful Life" actions, a new frontier of medical responsibility. J Matern Fetal Neonatal Med. 2013 Oct 31.

9. Devisch I. The tribunal of modern life: the case of UZ Brussels in the light of Odo Marquard's discussion on autonomy and theodicy. J Eval Clin Pract. 2013 Jun; 19(3):471-477. doi: 10.1111/jep.12042.

10. Manaouil C, Gignon M, Jardé O. 10 years of controversy, twists and turns in the Perruche wrongful life claim: compensation for children born with a disability in France. Med Law. 2012 Dec; 31(4):661-669.

11. Voelkerding KV, Dames SA, Durtschi JD. Next-generation sequencing: from basic research to diagnostics. Clin Chem. 2009 Apr; 55(4):641-658. doi: 10.1373/clinchem.2008.112789. Epub 2009 Feb 26. Review.
12. Jones D, Fiozzo F, Waters B, McKnight D, Brown S. First trimester diagnosis of Meckel-Gruber Syndrome by fetal ultrasound, with molecular identification of CC2D2A mutations by Next-Generation Sequencing. Ultrasound Obstet Gynecol. 2014 Apr 4. doi: 10.1002/uog.13381.

13. Łaczmańska I, Stembalska A. New molecular methods in prenatal invasive diagnostics. Ginekol Pol. 2013 Oct; 84(10):871-876.

14. Ansorge WJ. Next-generation DNA sequencing techniques. N Biotechnol. 2009 Apr;25(4):195-203. Epub 2009 Feb 3.

15. Shendure J, Ji H. Next-generation DNA sequencing. Nat Biotechnol. 2008 Oct; 26(10):1135g45.

16. Andorno R. The right not to know: an autonomy-based approach. Journal of Medical Ethics. 2004; 30(5):435-439.

17. Harmon A. Insurance Fears Lead Many to Shun DNA Tests. The New York Times, February 24, 2008

18. Driscoll DA, Gross S. Clinical practice. Prenatal screening for aneuploidy. N Engl J Med. 2009; 360:2556-2562.

19. Nicolaides $\mathrm{KH}$. Screening for fetal aneuploidies at 11 to 13 weeks. Prenat Diagn. 2011; 31: 7-15.

20. Wright CF, Chitty LS. Cell-free fetal DNA and RNA in maternal blood: implications for safer antenatal testing. BMJ. 2009; 339:b2451.

21. Syngelaki A, Pergament E, Homfray T, Akolekar R, Nicolaides $\mathrm{KH}$. Replacing the Combined Test by Cellfree DNA Testing in Screening for Trisomies21, 18 and 13: Impact on the Diagnosis of Other Chromosomal Abnormalities. Fetal Diagn Ther. 2014 Feb 8.

22. Position Statement from the Italian College of Fetal Maternal Medicine: Non-invasive prenatal testing (NIPT) by maternal plasma DNA sequencing. J Prenat Med. 2013 Apr; 7(2):19-20.

23. Benn P, Borell A, Chiu R, Cuckle H, Dugoff L, Faas B, Gross S, Johnson J, Maymon R, Norton M, Odibo A, Schielen $P$, Spencer K, Huang T, Wright D, Yaron Y. Position statement from the Aneuploidy Screening Committee on behalf of the Board of the International Society for Prenatal Diagnosis. Prenat Diagn. 2013 Jul; 33(7):622-629. doi: 10.1002/pd.4139. Epub 2013 May 21.

24. Gregg AR, Gross SJ, Best RG, Monaghan KG, Bajaj K, Skotko BG, Thompson BH, Watson MS. ACMG statement on noninvasive prenatal screening for fetal aneuploidy. Genet Med. 2013 May; 15(5):395-398.

25. American College of Obstetricians and Gynecologists Committee on Genetics. Committee Opinion No. 545 Noninvasive prenatal testing for fetal aneuploidy. Obstet Gynecol. 2012 Dec; 120(6):1532-1534.

26. Tjio JH \& Levan $A$. The chromosome number of man. Hereditas, 42, pag 1-6, 1956.

27. Langlois S, Duncan A. Use of a DNA method, QF-PCR, in the prenatal diagnosis of fetal aneuploidies. $\mathrm{J} \mathrm{Ob-}$ stet Gynaecol Can. 2011 Sep; 33(9):955-960. Review.

28. Maxam AM, Gilbert W. A new method for sequencing DNA. Proc Natl Acad Sci U S A. 1977 Feb; 74(2):560-564.

29. Sanger F, Coulson AR. A rapid method for determining sequences in DNA by primed synthesis with DNA polymerase. J Mol Biol. 1975 May 25; 94(3):441-448. 
30. Sanger F, Nicklen S, Coulson AR. DNA sequencing with chain-terminating inhibitors. Proc Natl Acad Sc U S A. 1977 Dec; 74(12):5463-5467.

31. Kallioniemi A, Kallioniemi OP, Sudar D, Rutovitz D, Gray JW, Waldman F, Pinkel D. Comparative genomic hybridization for molecular cytogenetic analysis of solid tumors. Science. 1992; 258(5083):818-821.

32. Faundes V, Pardo RA, Castillo Taucher S. Genetics of congenital deafness. Med Clin (Barc). 2012 Oct 20; 139(10):446-451. Epub 2012 Apr 24.

33. Ptok M. Early detection of hearing impairment in newborns and infants. Dtsch Arztebl Int. 2011 Jun; 108(25):426-431. Epub 2011 Jun 24.
34. Langfelder-Schwind E1, Karczeski B, Strecker MN, Redman J, Sugarman EA, Zaleski C, Brown T, Keiles S, Powers A, Ghate S, Darrah R. Molecular testing for cystic fibrosis carrier status practice guidelines: recommendations of the National Society of Genetic Counselors. J Genet Couns. 2014 Feb;23(1):5-15. Epub 2013 Sep 7.

35. L. 22 maggio 1978, n.194 - Norme per la tutela sociale della maternità e sull'interruzione volontaria della gravidanza.

36. Sentenza Corte di Cassazione n. 16754 del 2 Ottobre 2012

37. http://www.orpha.net/ 